\title{
BASIC CONCEPTS AND APPROACHES TO DEVELOP INTEROPERABILITY OF ENTERPRISE APPLICATIONS
}

\author{
David Chen ${ }^{1}$ and Guy Doumeingts ${ }^{1,2}$ \\ (1) LAP/GRAI, UMR CNRS 5131, University Bordeaux 1 \\ 351 Cours de la Libération, 33405 Talence FRANCE \\ Chen@lap.u-bordeaux.fr \\ (2) GRAISOFT, 33170 Gradignan, FRANCE \\ gdoumeingts@graisoft.com
}

\begin{abstract}
This paper aims at defining a basis to develop the interoperability of enterprise applications. After having stated our objectives in introduction, the paper will present the basic concepts and definitions relative to enterprise applications and interoperability. Then the three possible approaches to develop interoperability i.e. integrated, unified and federated scenarios are discussed in detail in order to clarify their underlying concepts. A model to develop interoperability in a federated environment is put forward. Future work is discussed as part of conclusions given at the end.
\end{abstract}

\section{INTRODUCTION}

With the globalisation of commerce, distribution and manufacturing, co-operation between enterprises of different sectors and cultures is significantly increasing. It is not only limited to sub-contracting and co-operation with suppliers and customers better known as 'supply chain' or 'extended enterprise', but also concerned with 'virtual enterprise' that can form and dissolve very quickly. There also exists a strong demand to preserve cultural identity and particular ways of working within the company. This leads inevitably to some interoperation situation rather than a tied integration. At a company level, the need on flexibility and reactivity, the inspiration of employees to work in a more autonomous way using their own method and tool, oblige an enterprise to change from traditional hierarchically based organisation to smaller, autonomous, distributed units structure. In this context, interoperability would occur in federated situation rather than total integrated one (Chen, 2002). Interoperability will become a permanent issue of research and experimentation since heterogeneity and constant change will persist for the foreseeable future.

The objective of the paper is to tentatively provide some basic concepts, approaches and clarifications so that the R\&D on the interoperability can be carried out in a better basis. Focus of the discussion is at a higher level of abstraction which 
is technology and industry sector independent. Standards will be adopted as possible as we can because they play an important role to make the interoperability happen.

\section{CONCEPTS}

\subsection{Enterprise application}

The term 'application' is usually misunderstood as a synonym of software. According to ISO 15745, an (manufacturing) application can be modelled as a combination of a set of processes, a set of resources, and a set of information structures that are shared and exchanged among the resources. In ENV 12204 (a European pre-standard), three types of resource have been considered: machining, computing and human types. Concrete examples of enterprise resources are communication networks, devices, software, equipment and personnel that are necessary to support the processes and information exchanges required by the application. An UML model of enterprise application, based on the definition of ISO 15745 and ENV 12204, is proposed as shown figure 1.

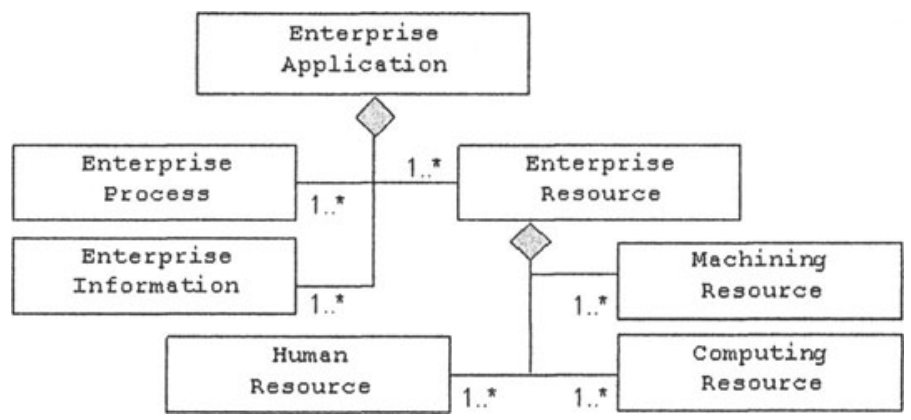

Figure 1 - An UML Model of enterprise application (adapted from ISO 15745)

\subsection{Interoperability}

There exist various definitions on interoperability. According to the Oxford Dictionary, interoperable means 'able to operate in conjunction'. The word "interoperate" also implies that one system performs an operation on behalf of another system. From software engineering point of view, interoperability means that two co-operating software systems can easily work together without a particular interfacing effort. It also means establishing communication and sharing information and services between software applications regardless of hardware platform(s).

Referring to the definition adopted in section 2.1, the interoperability of enterprise applications can be defined as the ability of interaction between the applications. The interoperability is considered achieved if the interaction can, at least, take place at the three levels: data, resource and business process with the semantics defined in a business context. The communication interoperability is considered as achieved with current technology. 


\subsection{Interoperability (relevant) domains}

According to the view hold in IDEAS project (IDEAS, 2002), developing interoperability of enterprise applications is concerned with the three research domains: (1) Enterprise modelling (EM), (2) Architecture/platform (A\&P), and (3) Ontologies (ONTO).

The Architecture/platform consists of the set of technology based services/functions which allow actually implementing and executing enterprise applications in a distributed inter or intra organisational environment.

The role of Enterprise Modeling vs. interoperability is to describe, when an application operates, what part of $E M$ is useful for communication, data, and process to interoperate with other applications.

Ontologies are to define the meaning of the terms used in a given business area and are used mainly to cover the semantic aspects of interoperability.

In other words, Enterprise Modelling and Ontologies are to model the part of business and knowledge that have an impact on the interoperability of enterprise applications i.e. the dependency of application interoperability on EM and Ontologies (Doumeingts et al., 2003).

\subsection{Interoperability framework}

The framework of interoperability aims at defining on the one hand various layers that are logically related, and on the other hand mapping the three domains (EM, A\&P and ONTO) to layers. It allows qualitatively evaluating the matter of concern between a layer and a domain (see table 1).

Table 1 - The framework of interoperability of enterprise applications

\begin{tabular}{|c|c|c|c|}
\hline & EM & A\&P & ONTO \\
\hline Process & +++ & + & ++ \\
\hline Resource & ++ & ++ & ++ \\
\hline Data & + & +++ & +++ \\
\hline Communication & + & +++ & + \\
\hline
\end{tabular}

The notation ' +++ ' means that a domain contributes significantly to a layer; ' + ' means that the contribution is weak; and ' ++ ' is in between. The relationship between the layers and domains is not 'linear'. Each domain contributes to all the layers but with different degree. The framework will also be used to identify more precisely the problems and solutions in each of the 'sub-area' of the table in order to develop the interoperability.

Generally speaking, interoperations occur between two or more resources (be they software, human or machine). It is resource that requests services to another resource. To establish the interoperation, data/information is exchanged through communication means and this according to a pre-defined (business) process. Data interoperability is the basic level, since any other level of interoperability requires a common understanding on data. 


\subsection{Enterprise modelling vs. interoperability}

Enterprise Modelling (EM) can be defined as the art of "externalising" enterprise knowledge, i.e. representing the enterprise in terms of its organisation and operations (e.g. processes, behaviour, activities, information, object and material flows, resources and organisation units, system infrastructure and architectures) (Doumeingts, 2003). Enterprise Modelling provides a semantic unification space at the corporate level where shared concepts can be properly defined, mapped to one another and widely communicated in the form of enterprise models (Goranson, 1992).

There are at least three roles played by enterprise modelling vs. interoperability of enterprise applications:

(a) Enterprise modelling to elaborating specifications to choose interoperable applications \& software on the market. In this case better model of commercial application/software is needed.

(b) Enterprise modelling to help establishing interoperability of between existing heterogenous applications \& software. In this case dynamic adaptation and parameterisation are required.

(c) Enterprise modelling vs. software engineering to elaborating specifications to develop interoperable applications. In this case better link between EM and application specifications is needed.

\section{APPROACHES}

Interoperability can occur in various organisational environments. The view from ISO 14258 is adopted to define the context of interoperations. Interoperation occurs between two (or more) entities (e.g. enterprise applications). There are three ways to relate them to one another (see table 2): (1) integrated; (2) unified; (3) federated. The federated situation is the most probable scenario for full interoperability wherein most models will not be in a standardised or common form because it is not economically feasible to put them in such a form (ISO 14258).

Table 2 - Three interoperability scenarios

\begin{tabular}{|l|l|l|}
\hline Paradigm & Description & Approach \\
\hline Integrated & $\begin{array}{l}\text { - Standard format for all models } \\
- \text { the format must be as detail as } \\
\text { models }\end{array}$ & $\begin{array}{l}\text { - Standardisation } \\
\text { - Mapping to the standard }\end{array}$ \\
\hline Unified & $\begin{array}{l}\text { - common format only exists at a } \\
\text { meta-level } \\
- \text { meta-model is not an executable } \\
\text { entity }\end{array}$ & $\begin{array}{l}\text { - Must have a pre-defined } \\
\text { meta-model for semantic } \\
\text { equivalence } \\
\text { - Mapping via meta-model }\end{array}$ \\
\hline Federated & $\begin{array}{l}\text { - No common format } \\
\text { - Dynamic accommodation }\end{array}$ & $\begin{array}{l}\text { - Must share an ontology } \\
\text { - Concepts mapping done } \\
\text { at ontology level }\end{array}$ \\
\hline
\end{tabular}


The view of ISO 14258 also allows to clarifying the difference between the concepts of integration and interoperability. Two integrated systems are necessarily interoperable; while two interoperable ones are not necessarily fully integrated.

Figure 2 gives an illustration of the integrated, unified and federated approach from eBusiness perspective (Man-Sze, 2003). In the case of unified approach for example, the common format that must exist could be 'ebXML' which actually acts as a metamodel.

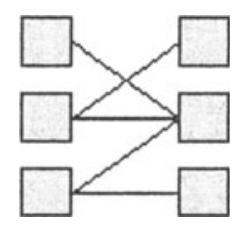

Integrated Approach

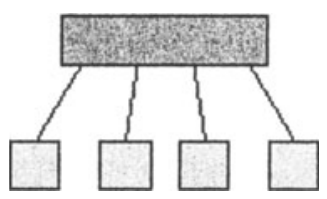

Unified Approach

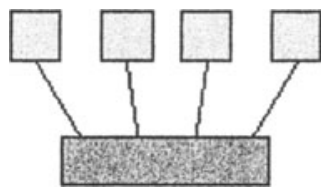

Federated Approach

Figure 2 - Illustration of integrated, unified and federated scenarios

\subsection{Integrated approach}

Designing fully integrated systems usually follows a top-down approach starting at the enterprise modelling level. Conceptual and business oriented modelling languages are used to represent user's requirements. The enterprise models are then transformed to more technology-dependent models allowing implementation of the designed system.

Since the years of 80 's, various integrated approaches have been developed in Europe and in USA. We can mention for example some well known approaches such as CIMOSA (Computer Integrated Manufacturing Open System Architecture) developed by AMICE consortium within the frame of the Esprit Programmes, GRAI Methodology elaborated at the LAP/GRAI of University Bordeaux 1, IEM (Integrated Enterprise Model) developed by IPK Berlin.

In CIMOSA, a set of modelling language constructs and associated templates were defined. Developing a CIMOSA system means that all system's components must be designed and implemented using CIMOSA constructs/templates. Divers models of the system can be described as detail as CIMOSA construct templates. Consequently, the system so designed can ensure the interoperability in priori.

The GRAI decisional approach is another example of integrated approach. It focuses on consistent and integrated enterprise-wide decision-making (Doumeingts, $1984,1998)$. Integration by decision means that the decisions made within various functions must be consistent in the sense that they must contribute to the achievement of the global objectives of the enterprise. For example, in the domain of production management, the decision for load-levelling is quite complex and requires a set of consistent decisions. Using a common format (for example the GRAI grid language), the decision system so designed should allow interoperations between various decision centres of the system. 
The integrated approach ensures the global consistency and coherence of the system. Various components of the system are designed and implemented using a common format (or standard) so that the interoperability is seen as a designed-in quality (of the system's components). Interoperation between various parts can be obtained without any supplementary interfacing efforts.

\subsection{Unified approach}

The unified approach applies in a heterogenous environment where divers existing models/systems were designed using different formats. To establish interoperability between these heterogenous elements using a unified approach, an agreement must be reached to define a common neutral format at a meta-level. Information can be exchanged by mapping divers models to the meta-model.

Some existing approaches which would fit within the unified scenarios are:

- STEP which aims at achieving interoperability between product data models.

- PSL (Process Specification Language) initially developed by NIST (National

Institute of Standards and Technology, USA) but now also moved into standardisation process.

The UEML project can be also considered for example as a unified approach to develop interoperability between enterprise models and tools. It has been considered that current software tools supporting enterprise modelling (such as $\mathrm{MO}^{2} \mathrm{GO}$, Metis and e-Magim supporting respectively the language IEM, IIEM and GRAI) were not interoperable. To achieve interoperability between them, a set of neutral enterprise modelling constructs were elaborated, called UEML constructs. These constructs constitute an agreed meta-model that divers enterprise models can be mapped on. To make interoperability happen, software developers must create the mapping between its own constructs to UEML constructs and implement the conversion as shown figure 3 .

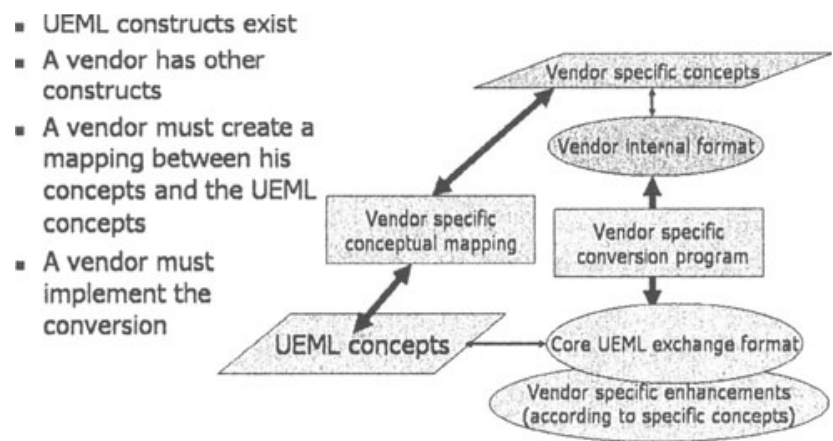

Figure 3 - Example of unified approach: the UEML initiative

In summary, with the unified approach a mapping mechanism at meta-level needs to be pre-defined. Standardisation is useful because it reduces the number of models to be mapped. 


\subsection{Federated approach}

The federated approach also applies in a heterogenous environment where divers existing models/systems were designed using respective proprietary formats. In a federated environment, no parties can impose their vocabularies, models and tools to others. To interoperate in such a context, instead of using the unified approach through a meta-model, dynamic accommodation and adaptation between parties are required. This implies that concepts mapping is done at an ontology level. The interoperability is then not a designed-in quality, but an added-on functionality.

Gusmeroli (2002) has explained that the federated approach (i.e. dynamic mapping) implies the existence of a "mapping factory" which is able to dynamically create the necessary mapping tools to inter-operate existing models without defining a meta-model infrastructure. New standards and even new models could this way be made compliant with existing state-of-the-art ones in a dynamic way. The role of semantics is fundamental here.

An example of establishing interoperability of enterprise applications between two companies in a federated environment is conceptually illustrated in figure 4.

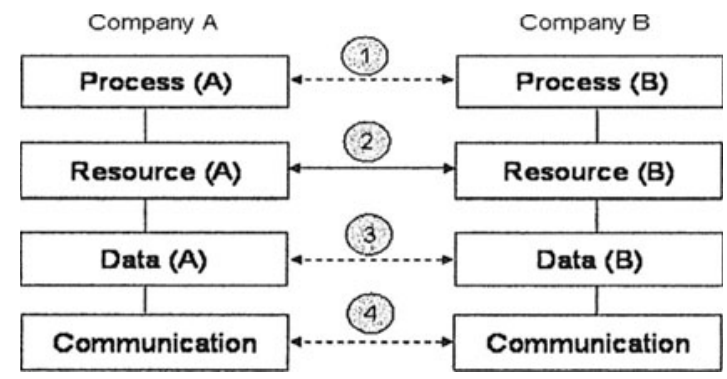

Figure 4 - Interoperation example using the federated approach (Illustration only)

The interoperation takes place between two resources (ex. software A and software B) to exchange services at the level (2). At first, request of interoperation will be issued from A to B (possibly by humans). A negotiation session between two parts allow $A$ and $B$ to map semantics from one to other and to exchange resource profiles as well as the desired result from $B$ and required input from $A$. The negotiation involves a dynamic accommodation and adaptation of the semantics at both sides in order to be mutually understood (2). At the (Business) process level, models of process A and process B are mutually exchanged (1) in order to determine and synchronise the interoperation actions. According to agreed semantics (if there is no shared ontology) and resource profiles exchanged, dynamic re-configuration and/or parameterisation might be necessary at the level of data (3) in terms data semantics and formats on the one hand, and on the other hand at the level (4) in terms of the communication protocols (4). The interoperability so established would allow the two resources to automatically exchange service (data B) at a given point of process $A$ by using data $A$ (as input to resource $B$ ) via communication means.

In summary, to achieve meaningful interoperation between companies, all levels of the enterprise are concerned. More concretely, we can say that the exigency for interoperability is born at the resource level (only resources perform works). It 
requires elements coming from both upper and lower levels and is not only a matter of data. An interoperability event passes through all the levels and is physically transferred via Communication (Connectivity and all the other ISO levels) and then can affect the destination enterprise at the all levels. The arrows in figure 4 represent a dynamic mapping mechanism which allows the four levels of both sides to adapt their syntax and semantics in such way so that information/services so exchanged can be correctly understood and used without any supplementary interfacing effort.

\section{CONCLUSION}

This paper has presented some basic concepts and approaches to develop the interoperability of enterprise applications. It also aimed at clarifying some confusing concepts/terms used in this domain such as integration vs. interoperability. Three possible interoperability scenarios (integrated, unified and federated) were therefore discussed. The originality of the approach is to combine the knowledge in three research areas contributing to develop the interoperability: enterprise modelling, architecture \& platform and ontology. The framework has put forward the fact that developing meaningful interoperability affects all levels of enterprise and not only the data. The work to be performed in a near future is to define precisely problems and solutions in each of the areas identified in the interoperability framework.

\section{REFERENCES}

1. Chen, D. and Vernadat, D., "Enterprise Interoperability: A standardisation View", Enterprise Interand-Intra Organisational Integration, Eds. K. Kosanke et al., Kluwer Academic Publishers, ISBN 1-4020-7277-5. October 2002, pp. 273-282.

2. Doumeingts, G. and Chen, D., Developing interoperability of enterprise applications and software An European IST Thematic Network project: IDEAS, Proc. of the $2^{\text {nd }}$ CENNET workshop, 2003 (to appear).

3. Doumeingts, G. and Kromm, H., "Industrial Challenges, Vision and Goals: Enterprise Modelling and Software Enterprise Application, Project internal note, 28-10-2002.

4. Goranson, H.T., "ICEIMT in Perspectives - 92 to 97", Enterprise Engineering and Integration: Building international consensus, Eds.: K. Kosanke and J. Nell, Springer, 1997.

5. Gusmeroli, S., "TXT 2nd Contribution to IDEAS Roadmap discussion", IDEAS TXT view-part II, internal note, 29/07/2002.

6. IDEAS Consortium, Thematic Network, IDEAS: Interoperability Development for Enterprise Application and Software - Roadmaps, Annex1 - Description of Work, 13-05-2002.

7. UEML Consortium, IST - 2001 - 34229, Unified Enterprise Modelling Language (UEML), Thematic Network, Annex 1, Description of Works, 2003-07-21.

8. Li, Man-Sze, Illustration of integrated, unified and federated approach from the eBusiness perspective, internal note, Brussels, February $14^{\text {th }}, 2003$.

9. ISO 14258, 1999, Industrial Automation Systems - Concepts and Rules for Enterprise Models, ISO TC184/SC5/WG1, 1999-April-14 version.

10. ISO DIS 16100 (2000), Manufacturing Software Capability Profiling, Part 1 - Framework for interoperability, ISO TC/184/SC5, ICS 25.040.01.

11. Open Group (2000), TOGAF: The Open Group Architecture Framework, Document No. 1910, Version 6, December.

12. Vernadat, F.B. (1996), Enterprise Modelling and Integration: Principles and Applications, Chapman \& Hall, London. 